\title{
La conservation et la valorisation de l'Herbier de Tournefort au Muséum national d'Histoire naturelle
}

The preservation and valorisation of Tournefort's Herbarium at the National Natural History Museum

Denis Lamy et Aline Pelletier

\section{OpenEdition}

\section{Journals}

Édition électronique

URL : http://journals.openedition.org/ocim/132

DOI : 10.4000/ocim.132

ISSN : 2108-646X

Éditeur

OCIM

Édition imprimée

Date de publication : 1 juillet 2010

Pagination : 19-26

ISSN : 0994-1908

\section{Référence électronique}

Denis Lamy et Aline Pelletier, « La conservation et la valorisation de l'Herbier de Tournefort au Muséum national d'Histoire naturelle », La Lettre de l'OCIM [En ligne], 130 | 2010, mis en ligne le 01 juillet 2012, consulté le 01 mai 2019. URL : http://journals.openedition.org/ocim/132 ; DOI : 10.4000/ ocim. 132 


\title{
La conservation et la valorisation de l'Herbier de Tournefort
}

\section{au Muséum national d'Histoire naturelle}

\author{
Denis Lamy et Aline Pelletier *
}

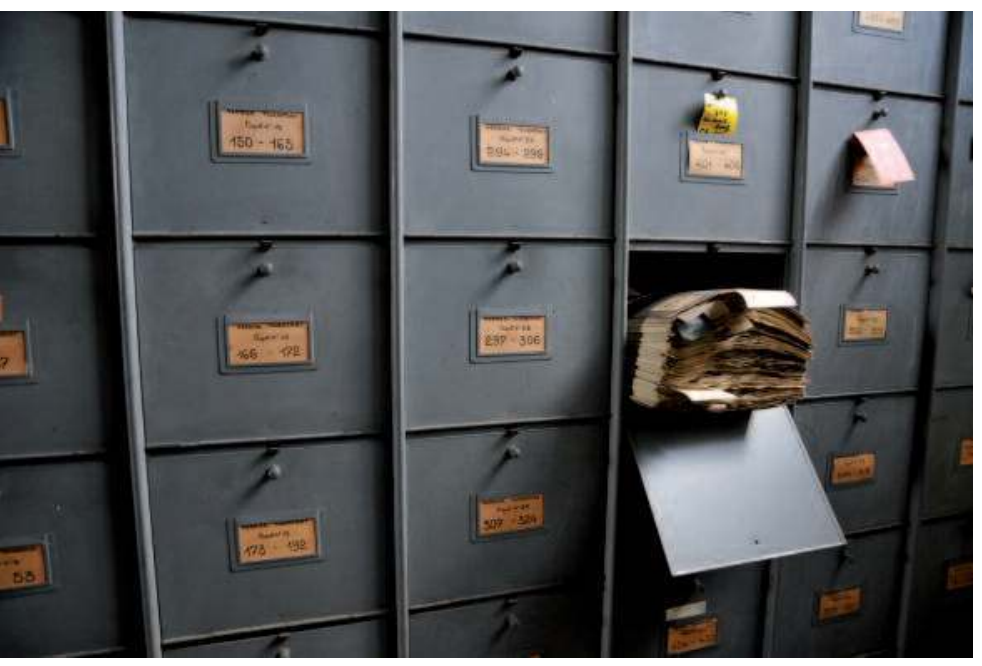

Détail d'un casier ouvert laissant entrevoir une liasse de I'Herbier de Tournefort, Herbier national, MNHN. (c) F. Bouazzat

* Denis Lamy est historien des Sciences au CNRS et responsable de la bibliothèque de Botanique du Muséum national d'Histoire naturelle lamy@mnhn.fr Aline Pelletier est élève conservateur territorial à l'Institut national du Patrimoine en Patrimoine scientifique, technique et naturel aline.pelletier@inp.fr
Constitué à la fin du XVIIe siècle, l'Herbier du botaniste Joseph Pitton de Tournefort présenté ici sert aujourd'hui - dans la perspective de la rénovation du bâtiment dit « de Botanique »- de modèle de travail pour l'étude menée au Muséum national d'Histoire naturelle sur la conservation et la valorisation des collections historiques de I'Herbier national et plus généralement pour la réflexion engagée autour de la place de la botanique au sein de l'établissement.

Au tout début du XIXe siècle, le botaniste français René-Louiche Desfontaines, titulaire de la chaire de «Botanique au Muséum » du Muséum national d'Histoire naturelle (MNHN) décide de former une collection générale de plantes sèches à partir des herbiers conservés dans le Cabinet du Roi et ayant appartenu à des savants attachés à l'institution créée en 1633. S'écartant de l'ancienne pratique de cabinet gardant intactes les collections privées, ce moment constitue une rupture. Desfontaines fusionne les herbiers entre eux et les classe par familles de plantes. Ainsi naît l'Herbier de Paris. Néanmoins, certains herbiers comme celui de Joseph Pitton de Tournefort (1656-1708) n'y sont pas intégrés pour des raisons historiques et/ou scientifiques.

Bien que classé Grand établissement scientifique et culturel, le MNHN ne présente aujourd'hui aucune exposition mettant en valeur l'Herbier, comptant près de dix millions de spécimens, et ce malgré la mission de médiation qui est attachée à son titre. Le développement de la Zoologie, rendu officiel en 1793 par la création de quatre chaires semble avoir 


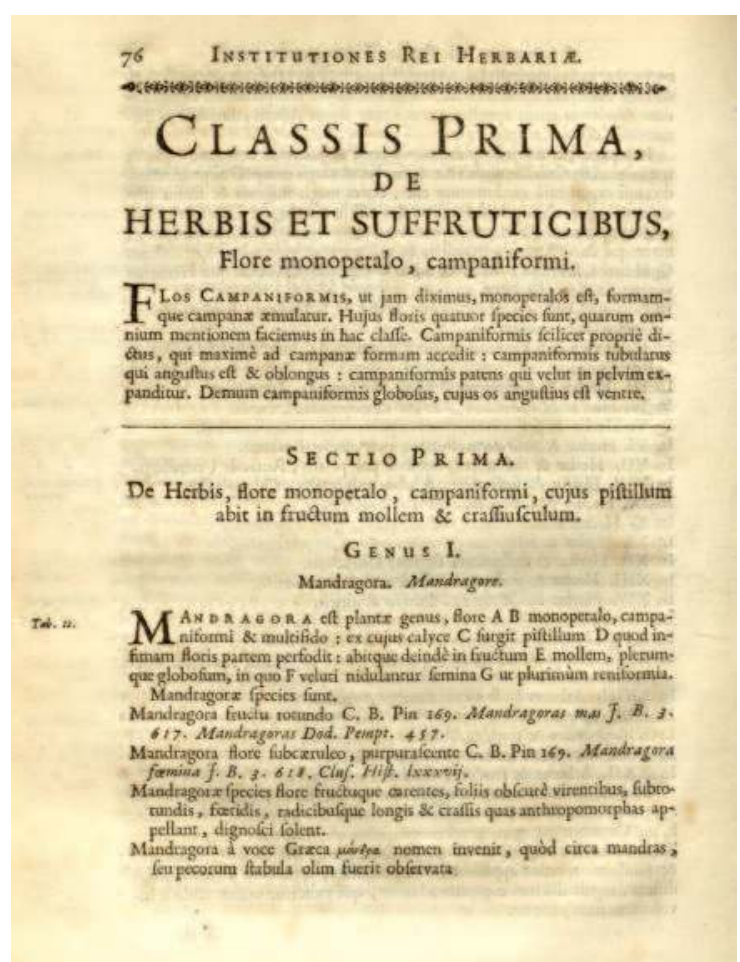

Frontispice du volume 3 des Institutiones de Tournefort (c) F. Bouazzat

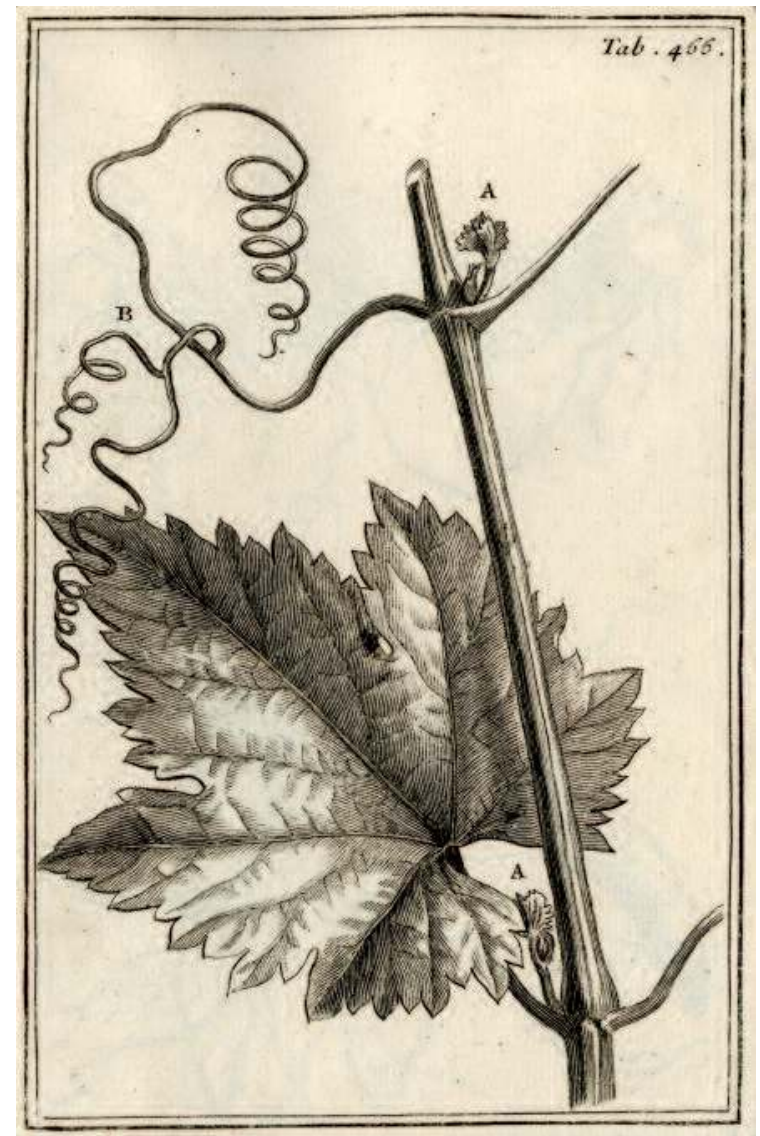

Vitis, planche gravée d'Aubriet mettant en avant le détail de la structure de la feuille et des vrilles, in Tournefort, Institutiones, volume 3 planche 466. placé la Botanique en arrière plan. La Grande Galerie de l'Évolution qui présente essentiellement des animaux naturalisés ou des squelettes témoigne de la difficulté à exposer le végétal qui attire moins que les collections d'animaux.

Pour autant, les récents travaux commencés en juin 2009 pour restructurer le bâtiment dit « de Botanique » abritant l'Herbier de Paris, ont permis de rouvrir le débat sur la valorisation de cette collection et sa présentation au public. C'est à ce titre que l'étude de l'Herbier de Tournefort prend tout son sens ${ }^{(1)}$.

\section{La conservation de la collection de plantes sèches de Joseph Pitton de Tournefort dans I'Herbier du MNHN}

\section{L'Herbier de Paris}

L'Herbier, conservé dans des rangées de casiers métalliques, est réparti sur trois étages. Une carpothèque (collection de fruits) et des collections d'artéfacts (peintures, moulages) accompagnent cet ensemble. On peut citer les cires de Robillard d'Argentelle (1777-1828), conservées tant pour leurs intérêts esthétiques que pédagogiques, ou les 540 champignons en cire du céroplasticien André Pierre Pinson (1746-1828) (2).

Les gestionnaires de l'Herbier sont des scientifiques qui portent le titre de chargé de collection. Selon le témoignage de Cécile Aupic, chargée des « herbiers historiques ", le statut de ce vaste ensemble reste flou. Une "note de service portant règlement des collections » de la direction des Collections du Muséum du 23 avril 2009 tente de clarifier les missions du MNHN : "Les collections sont utilisées à des fins de recherche et d'expertise, d'éducation et de délectation. Le Muséum est chargé de leur conservation, de leur étude, de leur enrichissement et de leur présentation au public ». Qualifiée de publique et nationale, la majeure partie de la collection de l'Herbier de Paris appartient au domaine public de l'État qui s'engage alors à en garantir la conservation pour la postérité ${ }^{(3)}$. Néanmoins, de par son intérêt biologique, cette collection doit rester disponible pour la recherche scientifique, ce qui signifie pouvoir offrir la possibilité de procéder à des prélèvements sur les spécimens. La gestion de l'Herbier du MNHN se doit d'être mieux définie car les catégorisations sont indispensables pour mener à bien des projets d'exposition pour des objets d'Histoire naturelle protégés par le Code du Patrimoine et par les lois sur la protection de la Biodiversité. 


\section{Les herbiers historiques}

L'Herbier du MNHN comprend d'un côté la collection générale qui, d'abord organisée en grandes régions du monde, répartit les végétaux par familles, genres et espèces, et de l'autre, la collection des herbiers historiques composée des collections présentant un intérêt historique et/ou scientifique. Le choix de conserver l'intégrité d'une collection particulière ou de la répartir dans l'herbier général s'explique par l'histoire de cet Herbier national. Lors de sa mise en place par Desfontaines, les herbiers conservés dans le Cabinet du Roi étaient ceux de Tournefort, de Sébastien Vaillant et de quelques petites collections. L'Herbier Tournefort, rangé selon sa méthode décrite dans son ouvrage majeur : les Institutiones rei Herbariae (1700), sera conservé intact et prendra l'appellation d'Herbier historique. Celui de Vaillant ne semblant pas refléter un ordre précis sera redéterminé et intégré à la collection générale.

Cet ensemble n'a aucun statut particulier au regard des lois sur le patrimoine. Cette ancienne désignation provient du traitement particulier envers ces

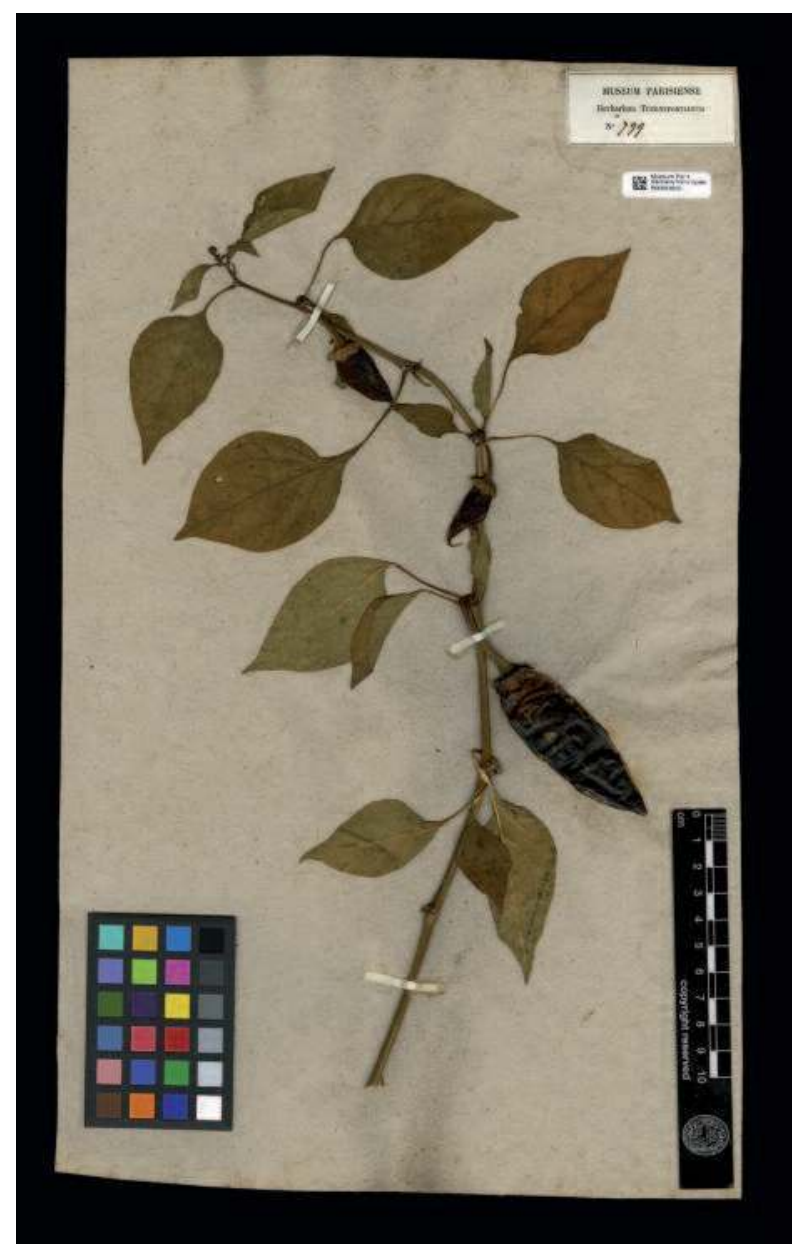

Capsicum, Herbier de Tournefort, P 00651822 , Herbier national, MNHN (c) L Kervran collections. Selon les mots de Cécile Aupic, « nous les [les herbiers historiques] protégeons bien sûr en raison de leur valeur historique et scientifique, c'est pour cela qu'ils ne sont jamais prêtés pour étude mais il s'agit d'un règlement intérieur et cela ne relève pas d'une loi particulière " (communication personnelle C. Aupic, 2009).

\section{L'Herbier Tournefort}

Il occupe 40 casiers dans lesquels sont renfermées des liasses, sortes de chemises cartonnées resserrées par une sangle. Dans chaque liasse, quelques dizaines de planches d'herbier sont rangées dans des dossiers suivant les vingt-deux classes (dix-sept d'herbes et cinq d'arbres) qui rassemblent 8000 espèces en 673 genres définis par Tournefort dans son ouvrage, les Institutiones. La collection apparaît ainsi comme une des formes d'application de sa méthode. En 1907, le botaniste Henri Lecomte évalue la collection à 6480 espèces réparties dans 700 genres et formant 68 paquets (Lecomte, 1907, n²2, pp. 679-680). Pour autant, rien ne permet d'affirmer

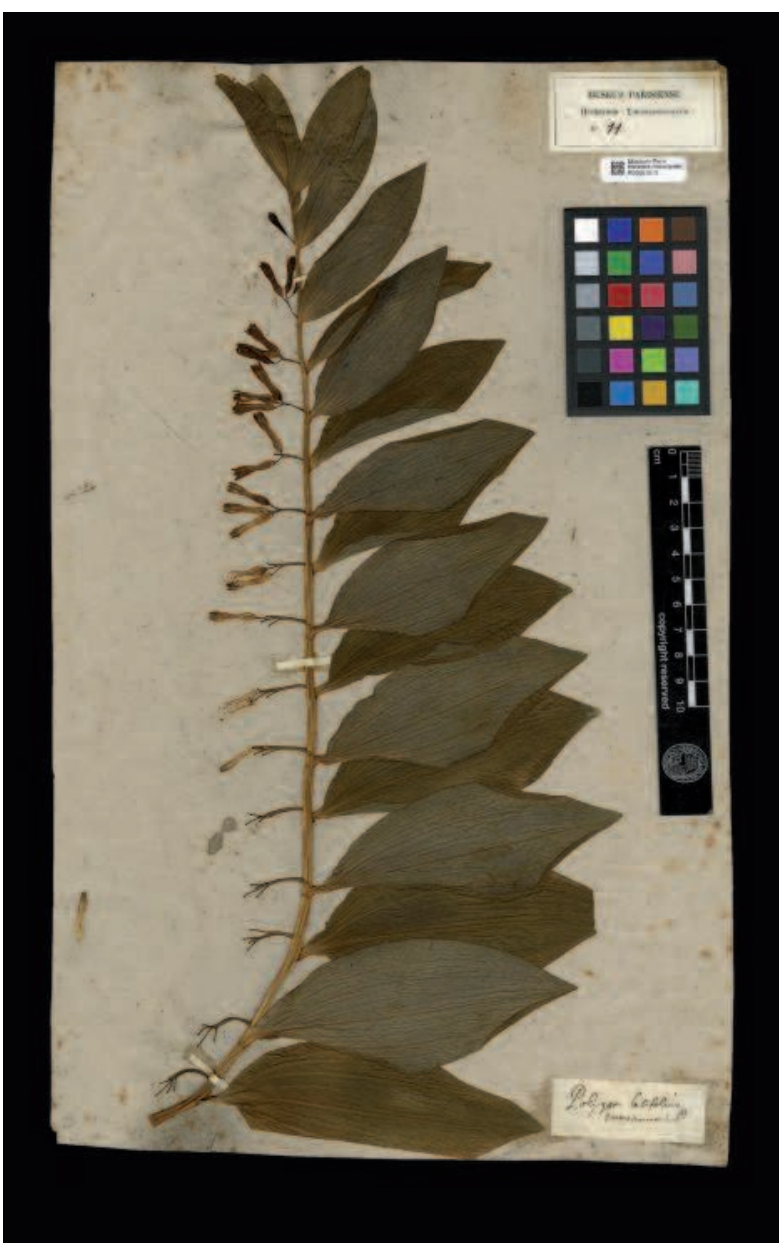

Polygonum, Herbier de Tournefort, P 00651013 , Herbier national, MNHN (). Kervran 
que la collection n'ait pas subi de changements entre cette évaluation et aujourd'hui, ni même depuis son legs par Tournefort.

\section{Formation de la collection}

À son arrivée à Paris en 1683, Tournefort a déjà une grande pratique de l'herborisation, que ce soit aux environs d'Aix, ou de Montpellier où il est inscrit à l'université de Médecine. Il herborise également dans les Pyrénées et en compagnie du Père Plumier, botaniste connu pour ses voyages en Amérique du Nord. Cette pratique est largement mise en œuvre lors des missions ordonnées par Fagon, Intendant du Jardin du Roi, dans le Midi de la France (1685-1687), puis en Angleterre, en Espagne et au Portugal. Une des prérogatives de Tournefort au Jardin du Roi est de fournir l'institution en nouvelles espèces pour les cultiver à des fins d'usages pharmaceutiques.

En 1700, sur ordre de Louis XIV, Tournefort part pour l'Anatolie, accompagné d'un dessinateur, Claude Aubriet (1665-1742), et d'un médecin allemand, Andreas von Gundelsheimer (1668-1715). Le but de cette mission est de reconnaître des plantes du Levant indiquées par Dioscoride (40-60 après J.-C.), médecin, pharmacologue et botaniste grec, d'en découvrir de nouvelles, mais aussi de faire des observations et des relevés de terrain pour la connaissance de la géographie et des coutumes de cette région. Le De Materia medica rédigé par le médecin antique fait, encore au XVIII ${ }^{e}$ siècle, autorité dans la connaissance des plantes médicinales du bassin méditerranéen et Tournefort le citera à maintes reprises dans sa Relation du voyage au Levant. Durant les deux années que dure l'expédition, il envoie à Paris des paquets remplis de graines ou de plantes séchées à l'attention de Fagon, de Sébastien Vaillant qui, de formation médicale, a suivi les cours de botanique de Tournefort, et de l'abbé Bignon, académicien, à qui il doit rendre compte de son voyage. Ainsi, dans une lettre qu'il lui adresse en juin 1700 , il lui remet " trois [plantes] de chaque espèce [...] E si à mon retour vous voulez m'en donner une [...] car il est fort malaisé d'en sécher beaucoup» (4). Ces longues périodes l'éloignent certes du travail du cabinet à Paris, mais cela lui permet d'observer et de récolter de nombreuses espèces nouvelles qui contribuent à compléter son herbier. Surtout son office de sous-démonstrateur de l'extérieur des plantes et la prise en charge de l'activité de l'Intendant Fagon lui permettent d'aborder la totalité des activités liées à la Botanique : des herborisations aux préparations pharmaceutiques, en passant par les cours publics de démonstration des espèces vivantes et le développement des cultures dans le jardin.

\section{Trois siècles de conservation : du cabinet privé de Tournefort aux galeries du Muséum}

Tournefort possède un cabinet privé dont le contenu est connu grâce à l'inventaire après décès dressé un mois après sa mort, le 31 janvier 1709. Son herbier qu'il nomme « jardin sec » (Brice, 1698, p.14), y est

\section{Essai sur l'origine de la collection de plantes sèches du Muséum}

Lorsque Tournefort rédige son testament, il fait le choix de léguer l'ensemble de sa collection d'objets d'Histoire naturelle au roi Louis XIV et ainsi de transférer sa collection à la sphère publique.

Cette pratique est loin d'être une habitude parmi les savants qui ont une charge au Jardin du Roi au début du XVIII siècle. La collection royale, montrée dans la galerie d'Histoire naturelle du château, s'est en réalité constituée selon des modes d'acquisition (achat, don, legs) dont aucun ne s'est systématisé et qui relèvent d'opportunités. À titre d'exemple, l'herbier de Sébastien Vaillant (1669-1722), premier garde du Cabinet du Roi, a été acheté à sa veuve en 1722, tandis que certaines collections importantes ont échappé à l'institution à l'image de l'herbier du jardinier André Thouin (1747-1824) acquis par Jacques Cambassedes (1799-1863) et aujourd'hui conservé à Montpellier. De manière générale, l'usage privé des collections constituées par l'argent public est une pratique ancienne et beaucoup de spécimens de collections n'entrent pas dans le cabinet du Jardin du Roi et se retrouvent sur le marché et alimentent les collections privées (Daugeron, 2009, pp. 58-62).

En outre, il est souvent difficile de savoir ce que l'on désigne sous le terme de «collections », un herbier ? Des herbiers ? Et de qui elles proviennent. Cette ambiguïté est entretenue dans les documents concernant les herbiers de l'institution, comme dans le Plan de travail établi par le naturaliste Lamarck (17441829) pour la mise en ordre des herbiers : « la totalité des plantes qui appartiennent au cabinet d'Histoire naturelle, c'est-à-dire avec les différents herbiers particuliers qu'il contient ou qui doivent s'y trouver » (5).

Si ces informations de provenance manquent souvent, le contenu exact de ces collections l'est plus encore. Alors que dans les documents d'archives du Muséum les collections zoologiques sont inventoriées spécimen à spécimen, les plantes le sont rarement voire jamais. 
rangé dans un mobilier adapté à la forme de conservation voulue : à plat, dans des tiroirs. Les notaires le décrivent placé dans quatre armoires « remplies de plantes sèches chacune dans une feuille de papier gris » (6). Germain Brice, auteur de guides de Paris de 1685 à 1752, a eu l'occasion de visiter ce cabinet. Il précise que les plantes sont «collées sur des feuilles de papier très proprement, avec leurs noms » (Brice, 1698, p. 14) ou «attachées » (Brice, 1706, tome 2, p. 18). Tournefort ne semble donc pas réticent à ouvrir les tiroirs pour des amateurs comme Germain Brice et encore moins pour des savants comme Martin Lister, naturaliste anglais qui qualifie sa collection de plantes sèches comme un des «plus beaux herbiers d'Europe» (Lister, 1873, pp. 66-68).

Placé dans le Cabinet d'Histoire naturelle du Roi en 1709, l'Herbier Tournefort devient un « trésor », rangé à la verticale dans la "bibliothèque » composée "des meilleurs livres [...] ainsi que les herbiers composés de 14000 plantes desséchées, E qui ont été recueillies par Messieurs Tournefort \& Vaillant » (Dézallier d'Argenville, 1742, p. 198). Cette situation a sans doute perduré jusqu'à la fin du XVIIIe siècle, au moment de la redistribution de l'ensemble des collections entre les trois règnes (animal, minéral et végétal). Ainsi, le 18 septembre 1793, une séance du Muséum d'Histoire naturelle prévoit que dans la quatrième pièce consacrée au règne végétal, "les livres ainsi que les herbiers seraient placés dans la grande salle $d u$ fond ${ }^{(7)}$. Les collections de plantes sèches accompagnent toujours les ouvrages.

Pour faire face à l'accroissement des collections (acquisition des collections laissées par le savant Réaumur, achat des collections rapportées du Sénégal par le voyageur-naturaliste Adanson) (Lamy, 1930, pp. 44-45), une habitation appelée « maison Léger », proche du labyrinthe du jardin, est acquise en 1795 par le tout nouveau Muséum d'Histoire naturelle. L'administration s'installe au rez-dechaussée laissant les étages libres pour entreposer les collections d'Histoire naturelle que l'on ne peut pas exposer dans les galeries du château. Le premier étage sert de dépôt à la Zoologie, le second à la Minéralogie et le troisième à la Botanique (8) où est placée la collection de plantes sèches de Tournefort.

Quarante ans plus tard, en 1836, une nouvelle galerie est destinée à accueillir du public avec la présentation des collections végétales au rez-de-chaussée et un premier étage dédié à la recherche et uniquement accessible aux scientifiques. Cet espace rassemble ainsi les conditions nécessaires à l'étude : bureaux de travail, bibliothèque, laboratoires et des collections non exposées au rez-de-chaussée. L'ensemble de la collection végétale est de ce fait partagé entre l'exposition et l'étude. Dans cette distinction, la collection générale de l'herbier ainsi que des herbiers régionaux ne sont pas exposés mais rangés dans les espaces du premier étage dédié à l'étude (Robin, 2000, p. 21).

Avec le déménagement, un premier inventaire accompagne l'Herbier Tournefort ; un index alphabétique des genres (chaque genre porte un numéro) est rédigé en 1812 par Sébastien Gérardin, employé à partir de 1810 comme aide-naturaliste pour les chaires de Botanique. Cette numérotation «post-tournefortienne » ne permet pas de se repérer dans l'herbier, rangé selon la classification exposée dans les Institutiones. Un autre document, plus volumineux, terminé en 1861, vient compléter ce travail. À l'intérieur, chaque planche est numérotée selon son ordre dans l'herbier. À titre d'exemple, les quatre premières planches sont celles du genre «Mandragora »; premier genre de la première classe, "de herbis et suffruticibus », exposée dans l'ouvrage de Tournefort les Institutiones. Ce catalogue permet un va-et-vient entre le livre et la collection.

Un siècle plus tard, en 1935, un nouveau bâtiment destiné à recevoir les herbiers est construit dans la continuité spatiale, en direction de la Seine, du pavillon de 1836. C'est celui que nous connaissons aujourd'hui et qui abrite l'Herbier national.

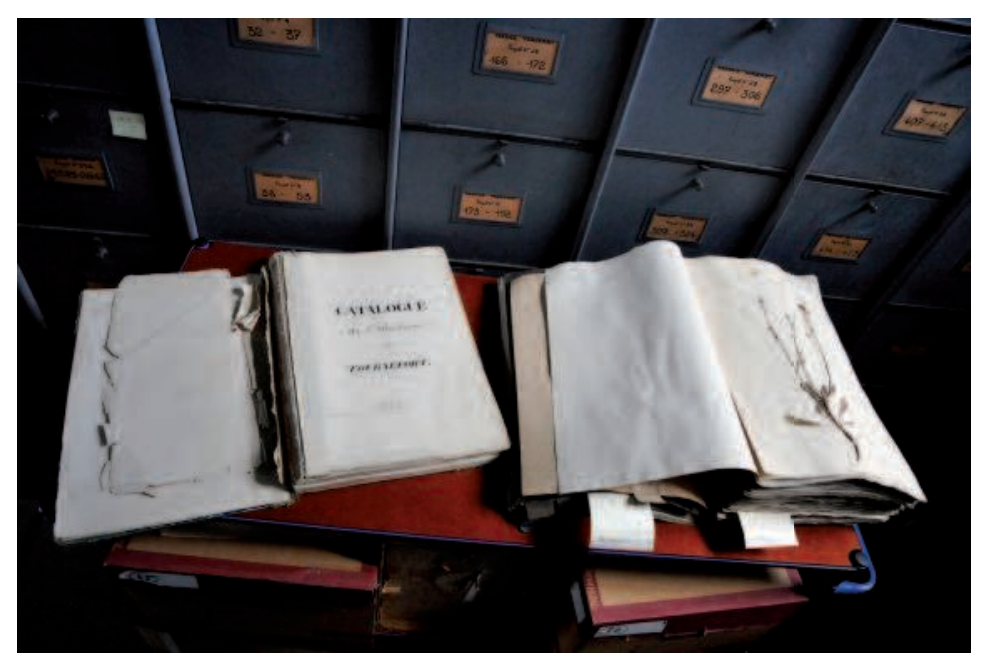

Vue d'une liasse ouverte et du catalogue de 1861, Herbier de Tournefort, Herbier national, MNHN (c) F. Bouazzat 


\section{Valorisation de l'herbier : axes de recherche}

\section{Comprendre les collections de botanique réunies de manière générale sous \\ le vocable d'Herbier de Paris}

La valorisation des collections ne passe pas seulement par une mise à niveau des spécimens dans leur présentation et leur accessibilité, elle nécessite aussi une réflexion historique. Cette réflexion est un des axes de recherche du groupe "Histoire de la Botanique : concepts et réseaux » au sein de l'équipe Botanique de l'Unité Mixte de Recherche 7205 (MNHN/CNRS) «Origine, Structure et Évolution de la Biodiversité ». Créé cette année pour une durée de quatre ans, ce groupe compte une douzaine de personnes et plusieurs projets. Pour appréhender une nouvelle approche des collections dites historiques et des collections en général de l'Herbier national, il a été décidé de considérer l'Herbier Tournefort comme un modèle de travail.

\section{Comprendre la place de la collection dans la pratique de Joseph Pitton de Tournefort}

L'étude en cours examine les différents liens que l'on peut tisser entre la pratique médicale de Tournefort, la pratique du jardin, l'enseignement, et la pratique de l'herbier, et ainsi, essayer de comprendre quel rôle Tournefort donnait à son herbier : une collection la plus complète des plantes connues, un simple aide-mémoire, ou un palliatif aux plantes absentes du jardin ?

Enfin, il s'agira de pointer les concordances entre les Institutiones et l'organisation de la collection. Cet ensemble de questions devrait permettre de dégager quelques conseils pour la conservation de cette collection.

\section{Évaluation et numérisation}

\section{des planches de l'herbier}

L'ensemble des taxons décrits par Tournefort dans les Institutiones a été saisi dans un référentiel taxonomique, permettant de retrouver l'histoire de chaque espèce par le jeu des synonymies. Les planches ont été numérisées et liées à ce référentiel. Il devient ainsi possible d'avoir une vue d'ensemble de la relation entre l'arrangement de l'herbier et l'ouvrage.

\section{Projet d'une galerie d'exposition dans}

\section{le bâtiment dit « de Botanique»}

Les grandes lignes de la rénovation du bâtiment sont :

- la restructuration des espaces de conservation de l'Herbier : installation de compactus et d'une climatisation.
La restauration des planches d'herbier (surtout de l'attache des parties végétales au support papier) a été confiée à la société GRAHAL ;

- la restructuration sur les quatre étages des espaces de recherche et de consultation de l'Herbier ;

- l'aménagement au rez-de-chaussée d'une nouvelle bibliothèque de Botanique réunissant les deux fonds préexistants (Phanérogamie et Cryptogamie) : imprimés et manuscrits. La collection de manuscrits et la collection iconographique seront correctement référencées, en lien avec les spécimens d'herbier;

- les architectes ont prévu le long de cette bibliothèque un espace d'exposition, côté rue Buffon.

Depuis 1935, la grande salle du rez-de-chaussée n’a en fait accueilli que très peu d'expositions consacrées à la Botanique, hormis les salons du champignon. Par ailleurs, ni la Grande Galerie de l'Évolution, ni le tout récent Cabinet d'Histoire installé au rez-de-chaussée de l'Hôtel de Magny n'évoquent ce thème pourtant historiquement important pour l'institution. De manière générale, la présentation de la Botanique reste un véritable défi en termes d'exposition du végétal et peu de muséums en France proposent des innovations dans ce domaine. Ce nouvel espace suscite de nombreuses propositions émanant de l'ensemble du personnel. D'après les plans des architectes, la galerie occuperait $80 \mathrm{~m}$ de long, $3 \mathrm{~m}$ de large, soit $258 \mathrm{~m}^{2}$. Outre les collections de plantes sèches, un patrimoine riche mérite d'être exposé au grand public : des artéfacts de plantes en cire ou en plâtre, des bustes, des documents iconographiques et des manuscrits conservés dans la bibliothèque qui longera la future galerie d'exposition. Parmi ces éléments, le dessin trouve une place toute particulière dans la transmission du savoir

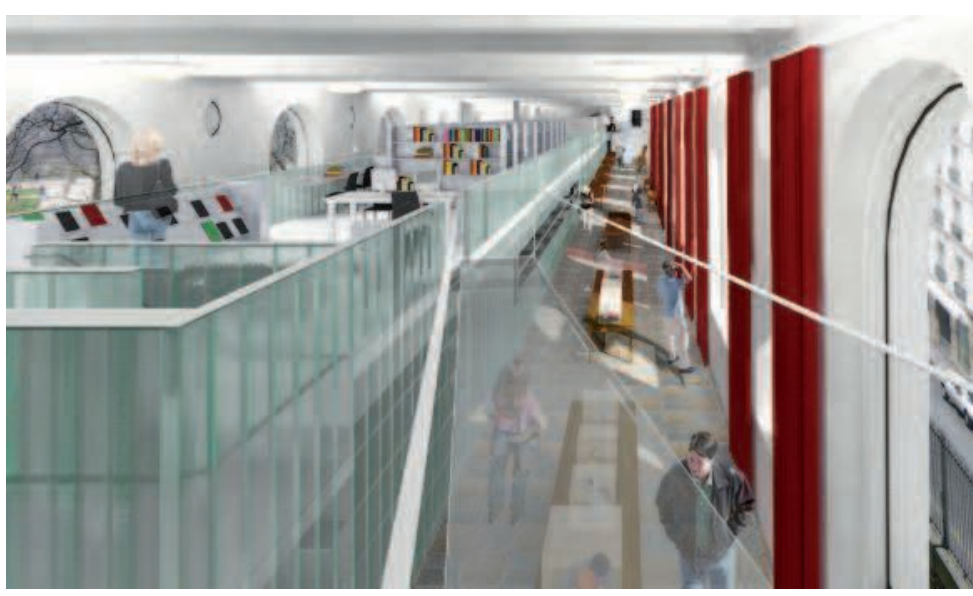

Perspective du rez-de-chaussée du futur bâtiment de Botanique (C) DR 
botanique (Lamy, 2008b, p. 139) car il n'est pas rare que des dessins soient les représentations exactes de planches d'herbier à l'image de ceux du Père Plumier publiés dans son Traité des fougères d’Amérique (1705) (Lamy, 2008b, p. 153).

La réussite de ce projet d'exposition réside dans l'articulation entre la richesse historique des collections et la présentation de la recherche contemporaine. La mise en valeur de cet ensemble mettrait en scène les concepts qui ont été et qui sont toujours au cœur de la pratique du botaniste. À titre d'exemple, la classification du monde végétal est un concept-clef qui a rythmé la recherche botanique depuis plusieurs siècles; ces systèmes reflètent une organisation de la Nature d'après différents botanistes. Peu à peu, ces systèmes se sont détachés du critère de l'usage de la plante au profit de critères morphologiques, physiologiques puis, plus récemment, moléculaires (Lamy, 2008a, p. 119).

Dans ce cadre, le siècle qui a vu naître Tournefort est tout à fait important. En effet, c'est au cours du XVII e siècle que la Botanique devient une véritable discipline qui cherche à classer méthodiquement toutes les plantes (Lamy, 2008a, p. 125). Ce tournant est également celui où la sexualité des plantes est de plus en plus admise dans la communauté des botanistes. L'Herbier Tournefort trouve toute sa place dans ce discours car il est un point de départ pour aborder la Botanique du XVIII e siècle, rythmée d'une part par les défenseurs des systèmes artificiels symbolisés par le système sexuel du suédois Carl von Linné (1707-1778) et, de l'autre, par les partisans de la méthode dite «naturelle », comme le botaniste de Jussieu au Jardin du Roi, qui cherche à mettre en évidence les relations entre les végétaux. Aujourd'hui, les classifications phylogénétiques tentent d'exprimer l'histoire de l'évolution des organismes végétaux (Lamy, 2008, p. 136).

Si à première vue l'espace prévu peut faire craindre des difficultés de présentation et de circulation, il convient néanmoins de tirer parti de la forme allongée de la galerie et de chercher à construire une " promenade botanique » allant des anciennes collections aux recherches actuelles. Dans ce cadre, la planche d'herbier constituerait une interface récurrente pour lier les diverses activités du botaniste qu'il soit contemporain ou qu'il ait vécu il y a plus de trois cent ans. À partir de l'Herbier Tournefort, plusieurs thèmes peuvent être abordés : la culture dans le Jardin des Plantes, le renouveau de l'école de Botanique, les expéditions et la création d'un réseau mondial d'échanges de plantes.

\section{Conclusion}

L'intérêt pour la science s'est considérablement accru ces dernières années, notamment avec l'émergence des problématiques environnementales. Les muséums dont la fonction de thésaurisation est essentielle, sont aujourd'hui concurrencés dans cette mission par d'autres établissements également voués à la diffusion de la culture scientifique. Mais par la singularité des collections d'Histoire naturelle qu'ils conservent, ils disposent d'un outil unique qu'ils peuvent utiliser pour développer des approches muséales novatrices.

Au Muséum national, l'Herbier de Joseph Pitton de Tournefort mais aussi l'histoire de la Botanique comme discipline à l'origine de la fondation du Jardin du Roi sont au final mal connus. Pourtant, l'étude qui a été entreprise ici nous montre la place essentielle des collections anciennes. Le caractère plutôt sévère d'une planche d'herbier doit alimenter le débat sur leur présentation auprès du grand public. La créativité et l'innovation dans le domaine de la médiation scientifique ont toute leur place dans la préparation d'une exposition sur la Botanique.

\section{Notes}

(1) Cet article reprend les réflexions exposées dans le mémoire de master 2 de Muséologie scientifique au MNHN d'Aline Pelletier, intitulé La collection de Joseph Pitton de Tournefort (1656-1708) au Muséum national d'Histoire naturelle : de la conservation de l'herbier à la découverte de la pratique d'un savant du début du XVIIIe siècle.

(2) Celui-ci s'est inspiré des planches imprimées en quatre couleurs du naturaliste Pierre Bulliard (1752-1793) dans son Histoire des champignons de France (1791-1792).

(3) L'appartenance au domaine public de l'État donne la qualité d'imprescriptibilité et d'inaliénabilité.

(4) Archives et manuscrits de la Bibliothèque centrale du Muséum (BCM), Ms 995 : " Mémoire que Monsieur de Tournefort [...] a envoiez [...] pendant son voiage en Orient, à Monsieur l'abbé Bignon [...] depuis le mois de mars 1700 jusques au mois de mai 1702 ». Les mémoires se composent de lettres, de catalogues et de descriptions de plantes, d'un journal de voyage... Copie par Morin.

(5) Archives nationales, CARAN, Série AJ/15, Archives du Muséum national d'Histoire naturelle, AJ/15/512 : pièce 503 : «Plan du travail à faire pour remettre en ordre les herbiers du Cabinet d'Histoire naturelle ", par Lamarck (24 avril 1793).

(6) Archives nationales, CARAN, Minutier central des Notaires, ET/ LVII/247.

(7) Archives et manuscrits de la BCM, Ms 2359. I : Varia, «notice historique sur les collections relatives au règne végétal $d u$ Jardin $d u$ roi et au Muséum depuis sa création et jusqu'en 1858 ». 
(8) Archives et manuscrits de la BCM, Ms 2359. I : Varia, «notice historique sur les collections relatives au règne végétal du Jardin du roi et au Muséum depuis sa création et jusquien 1858, [par mon père Adolphe Brongniart] ».

\section{Bibliographie}

Brice, G. Description nouvelle de la ville de Paris. Paris : chez N. Le Gras, N. Le Clerc, et B. Girin, 1698, 4e édition.

Brice, G. Description nowvelle de la ville de Paris. Paris : M. Brunet, 1706, volume 2 , 5e édition.

Daugeron, B. Collections naturalistes entre science et empire (1763-1804). Paris : Muséum national d'Histoire naturelle, Publications scientifiques, 2009, 635 p.

Dézallier d'Argenville, A.-J. Des plus fameux Cabinets d'Europe touchant l'Histoire naturelle, in Dézallier d'Argenville, A.-J., L'Histoire naturelle éclaircie dans deux de ses parties principales : la lithologie et la conchyliologie. Paris : Chez de Bure l'aîné, 1742, lère édition, pp. 198-230.

Lamy, D. Des classifications botaniques, in Allain, Y.-M. (et al.) Passions botaniques : naturalistes voyageurs au temps des grandes découvertes (exposition présentée au Domaine départemental de la Roche-Jagu, du 7 juin au 5 novembre 2008). Rennes : Édition Ouest-France, 2008, pp. 119 138. (Lamy, 2008a).

Lamy, D. Le dessin botanique dans la transmission des connaissances, in Allain, Y.-M. (et al.) Passions botaniques : naturalistes voyageurs au temps des grandes découvertes (Exposition présentée au Domaine départementale de la Roche-Jagu, du 7 juin au 5 novembre 2008). Rennes : Édition Ouest-France, 2008, pp. 139-155. (Lamy, 2008b).
Lamy, E. Les cabinets d'Histoire naturelle en France au XVIIIe siècle. Paris : E. Lamy, 1930, 58 p.

Lecomte, H. Historique des collections de Botanique du Muséum d'Histoire naturelle de Paris, Revue scientifique, 5e série, vol. 7, 1907, $n^{\circ} 22$, pp. 673-680 et $n^{\circ} 23$, pp. 716-721.

Lister, M. Voyage de Lister à Paris en 1698. Paris : Société des bibliophiles, Édition française, 1873, 347 p.

Pelletier, A. La collection de Joseph Pitton de Tournefort (1656-1708) au Muséum national d'Histoire naturelle : de la conservation de l'herbier à la découverte de la pratique d'un savant du début du XVIIIe siècle. Mémoire de Master 2 recherche "Muséologie, sciences, sociétés », Paris : MNHN, 2009, 139 p.

Robin, N. La présentation de la Botanique au Muséum national d'Histoire naturelle de 1793 à nos jours, entre barrières conceptuelles et obstacles muséographiques. Mémoire de DEA de muséologie des Sciences naturelles et humaines, Paris : MNHN, 2000, 94 p.

Tournefort, Joseph Pitton de Elémens de Botanique, ou méthode pour connoître les plantes. Paris : Imprimerie royale, 3 volumes, 1694, 3 vol., 410 p., 451 pl.

Tournefort, Joseph Pitton de. Institutiones rei herbariae. Paris : Imprimerie royale, 3 vol., 1700, [20]-697-[54] p., 489 pl.

Tournefort, Joseph Pitton de. Relation d'un voyage au Levant, fait par ordre $d u$ roi. Paris : Imprimerie royale, 2 vol., 1717. 